\title{
Diluting the dilution effect: a spatial Lyme model provides evidence for the importance of habitat fragmentation with regard to the risk of infection
}

\author{
Agustín Estrada-Peña \\ Department of Parasitology, Faculty of Veterinary Medicine, University of Zaragoza, Zaragoza, Spain
}

\begin{abstract}
This paper aims to construct a spatially-explicit model of Ixodes scapularis infection in the State of New York, USA, based on climate traits, high-resolution landscape features and patch-connectivity according to graph theory. The degree of risk for infection is calculated based on empirical data of host abundance, previous studies on host infectivity rates and tick preferences towards a given host. The outcome signifies what is called the "recruitment of infection", i.e. an index representing the abundance of infected ticks in a particular patch of vegetation. The results show that the I. scapularis recruitment of infection (IR) index is highly dependent upon a complex array of landscape fragmentation and the presence of key hosts. Neither faunal richness nor host density alone has any reasonable effect on the recruitment of infection. The experience of Lyme disease in the State of New York shows no clear relationship between the IR as calculated at the patch level and then summarized county by county and the rates of disease over the last eight years reported for these counties. However, areas characterized by low IR have consistently been associated with locations with low disease rates. Above all, the low levels of disease are related to minimal suitability due to the climate and negligible connection between patches. Social factors, mainly activities leading to an increased contact of humans with infective foci (which can be situated far from their homes), may lead to a high rates being reported from areas with high human densities rather than areas characterized by a high recruitment of infection. The spatial model developed here may be used to study the long-term changes in infective risk and tick recruitment as a result of humaninduced changes in the landscape.
\end{abstract}

Keywords: Ixodes scapularis, Lyme disease, recruitment of infection, habitat patchiness, connectivity.

\section{Introduction}

The human risk for Lyme disease in USA is dependent on the density of host-seeking Ixodes scapularis, i.e. the nymphal stage infected with Borrelia burgdorferi. However, this risk is further influenced by a complex relationship with climate, landscape characteristics and the number of available, adequate hosts. Attempts to map the distribution and the abundance of the tick vector have been

Corresponding author:

Agustín Estrada-Peña

Department of Parasitology

Faculty of Veterinary Medicine

University of Zaragoza

Miguel Servet 177, 50013-Zaragoza, Spain

Tel. +34 9767615 558; Fax +34976761 612

E-mail: aestrada@unizar.es overly simplistic as they are based only on wide-area climate information or on satellite imagery of land use and land cover without taking the hosts into account in the predictive models. Standardized efforts to measure the risk have been conducted at the state level, where the human risk for Lyme disease has been quantified only based on vector distribution (Drew et al., 1988; Novak et al., 1991; Kitron et al., 1992; Schulze et al., 1998; Guerra et al., 2002).

Ecological risk can be defined as the probability of exposure to an infection without active preventive measures. Risk-mapping with respect to Lyme disease is complicated by the fact that the distribution of I. scapularis seems to be expanding in some zones in USA (Madhav et al., 2004). Factors affecting the spatial position of pathogens, hosts and vectors, and 
their probability of close encounter, are fundamentally important to disease dynamics. Mapping of habitat suitability for ticks has a serious drawback regarding its use as proxy for Lyme disease as the presence of disease is rather more correlated with the presence of pathogen-infected vectors and adequate reservoir hosts than with the presence or abundance of ticks. Lyme disease in North America is largely restricted to the north-eastern and upper mid-western USA in spite of widespread populations of the tick vector in south-eastern and lower mid-western regions.

It is well-known that habitat type and fragmentation has a strong influence in the observed patterns of Lyme disease (Kitron et al., 1992). It has been also demonstrated that landscape context has a deep influence on the abundance of I. ricinus, an important tick in Europe (Estrada-Peña, 2003). The same effects have been demonstrated for I. scapularis (Madhav et al., 2004). These effects have been further modelled to show that tick abundance is correlated with patch shape and the degree of connectivity between high-quality patches for ticks, suggesting that host movements strongly influence tick abundance (Estrada-Peña, 2003). The effect of host diversity and community composition is also agreed to be a key component in the epidemiology of Lyme disease (Ostfeld and Keesing, 2000). We further demonstrated (Estrada-Peña et al., 2008) how the so called "dilution effect" in a tick-borne pathogen (Anaplasma marginale) can be difficult to link with models within complex landscapes supporting different densities of primary hosts (deer) and "diluting" hosts (wild boars).

It has been proposed that the incidence of Lyme disease could have a simple predictor, for example weather factors, mice abundance or acorn production (Schauber et al., 2005). However, it has been shown that Lyme disease foci are the result of complex interactions between a high number of components (Randolph, 1998) rather than a single piece of information, or a set of simple variables. In addition, the geographical distributions and densities of many arthropod vector species are sensitive to the climate because they are subject to ambient conditions during the non-parasitic part of their life cycles. Classification of habitat types could therefore be helpful in providing indicators for tick distributions by acting as proxy for tick survival (Guerra et al., 2002), thereby contributing to the development of risk maps. This paper addresses this question by integrating our current knowledge of the effects of habitat fragmentation to tick dynamics into a model which is applied to an entire state of USA. The approach is further used to derive the sensitivity of the system from changes in the range of some descriptor variables.

It seems that an explicit spatial model for Lyme disease risk could be reasonably well derived by rigorous definition of the absolute risk of a given area involving the tick habitat (climate-derived) suitability, the effects of landscape structure on tick abundance and the host community composition. The goal would be to produce a model accounting for changes in risk for infection by $B$. burgdorferi through the incorporation of climate effects upon tick habitat suitability, the effect of landscape patterns on tick abundance, and existing data on preferences of ticks towards defined hosts and their ability to infect the tick population. This model would not only provide a visual assessment of the high-risk areas due to high densities of infected ticks but also show that habitat fragmentation affects the risk index as much as host composition.

\section{Materials and methods}

\section{Background}

Previous work on the effects of the landscape on tick abundance (Estrada-Peña, 2003) resulted in the development of a recruitment index based on habitat patchiness, which was shown to be directly correlated with abundance of nymphal ticks. This was successfully tested for I. ricinus in northern Spain where a model of spatial abundance was linked with empirical data about the reservoir competence of wild hosts for the ticks and with data on spatial 
abundance of these hosts. This time, the approach has been used to provide an entomological index of I. scapularis ticks infected with Borrelia spp. in the State of New York, where high rates of Lyme disease in humans have been reported for many years (http://www.health.state.ny.us/statistics/chac/general/lyme.htm). This model explicitly lacks a seasonal component and is only meant to be a framework to better understand the identification of areas where there is a high risk for infected ticks. Figure 1 shows the study area.

A three-step approach has been used to construct the model. The first step requires the calculation of an index of climate suitability (CS), a parameter aimed to mark the adequacy of the climate for tick populations for the long term. This index adheres to the strategy of climate modelling developed for several arthropod species (i.e. Kalluri et al., 2007). The second step evaluates the effects of the landscape on the tick population based on a previously developed model of landscape fragmentation and its effects on tick abundance. The third step involves the calculation of an index of recruitment of infection (IR) based on the abundance of a range of hosts, their ability to feed different tick populations and to infect these ticks with Borrelia spp., as reported by Giardina et al. (2003) and LoGiudice et al. (2003). As this model is primarily intended to be used for the long-term estimation of risk along the spatial dimension rather than along the temporal one, the strong influence of the seasonal component on actual tick infection rates and the delicate tick/hosts dynamics are not considered here.

\section{Climate suitability (CS)}

A total of 1,759 I. scapularis records with adequate reference to spatial coordinates, originally selected from museum collections and published reports for a previous report on tick distributions

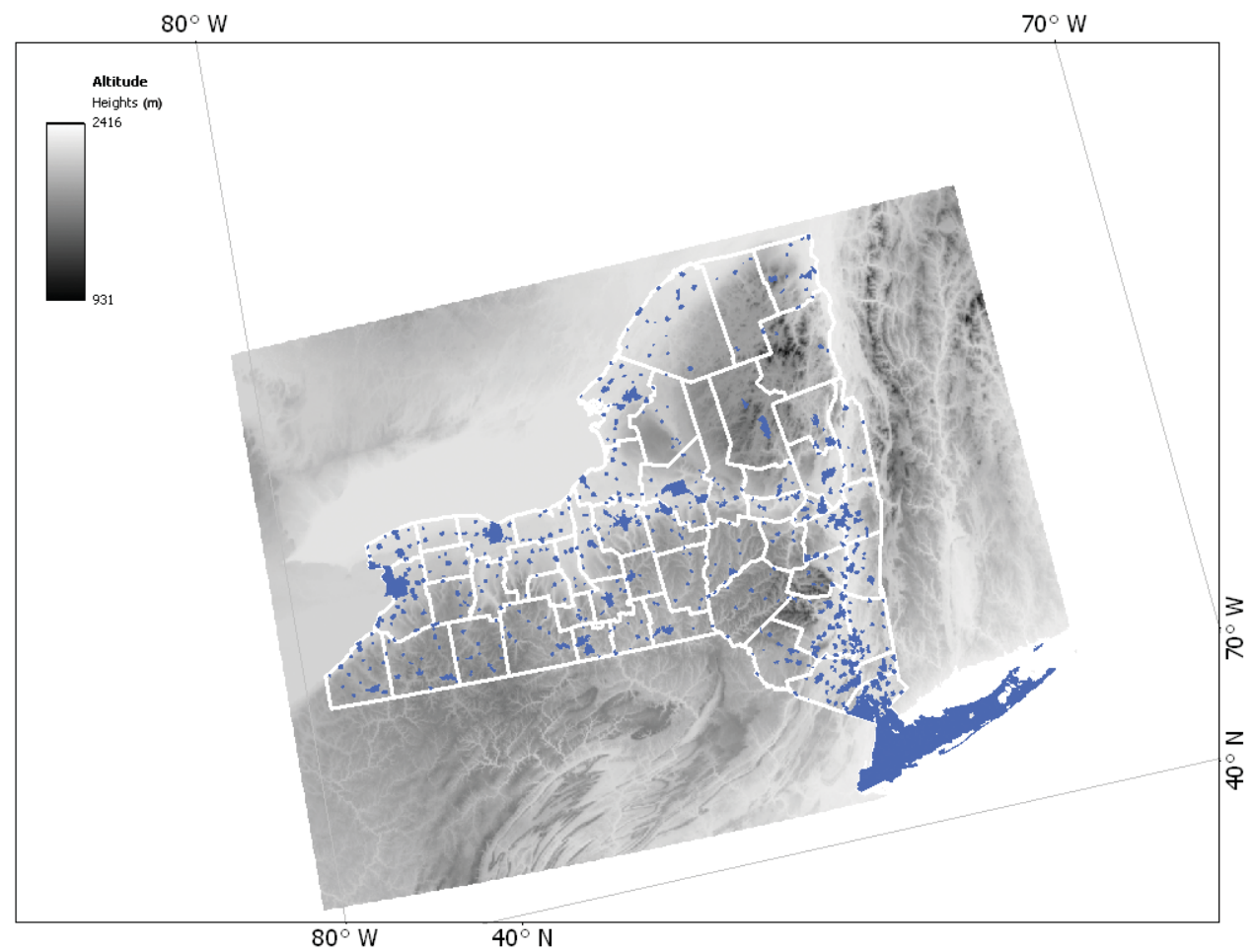

Fig. 1. The area of study in the State of New York, USA. The contours of the counties are delineated over a digital elevation model of the area. Coordinates showing latitude and longitude are included for localization purposes. Blue signifies urban areas. 
(Estrada-Peña, 2002), were used for the CS calculations. This data-set was updated with records from the U.S. National Tick Collection (http://www.bio.georgiasouthern.edu/iap/Tick_collec.htm). Additional records were obtained from local or regional surveys in USA through PubMed (http://www.ncbi.nlm.nih.gov/pubmed/) using the search term "Ixodes scapularis". This latter search provided a high number of papers not devoted to tick records, but also ensured that most reports in the PubMed database were included. Only adequately georeferenced data were used (i.e. referred to the name of a population unambiguously found in local gazetteers or providing pairs of coordinates). The maximum entropy algorithm (Phillips et al., 2006) was used to model the distribution of presence-only records against a set of raster environmental layers. The climate data-set used, obtained from the PRISM model (http://www.prism.oregonstate.edu), included records collected between 1980 and 2007 on the monthly minimum and maximum temperatures and the accumulated monthly rainfall. Models were developed with a random training set consisting of $50 \%$ of the total tick records and checked against the remaining $50 \%$ of the total number of tick records. The performance evaluation of the model was calculated using the area under the curve (AUC) of a receiver operating characteristic (ROC) plot of sensitivity against (1-specificity) (Swets, 1988).

\section{Estimation of recruitment values from landscape structure}

The model provides a spatial index of recruitment, directly related to the density of ticks at the patch level, based on the previous CS and a network of patches. Network properties and mathematical structure have their roots in seminal works by Urban and Keitt (2001). Networks of habitat patches have been widely relied upon to better understand the movements of animals according to the properties of the landscape and to model the infection rates of wild animals with tick-borne pathogens (Estrada-Peña et al., 2008). This model has been exhaustively validated for I. ricinus based on empirical data (EstradaPeña et al., 2004). No attempts were made to validate the model for I. scapularis, and it is assumed that factors regulating the spatial distribution of the recruitment index operate at the same level. According to graph theory (see Urban and Keitt, 2001), the probability that an individual in node $i$ will disperse to node $j$ can be expressed as a flux rate or a dispersal probability matrix. Thus, the expected dispersal flux from patch $i$ to patch $j$ is:

$$
f_{i j}=\frac{S_{i}}{S_{t o t}} p_{i j}^{\prime}
$$

where $S_{i}$ is the area of patch $i, S_{t o t}$ the sum of the areas of every available patch, and $p^{\prime}{ }_{i j}$ the probability of dispersal from $i$ to $j$. This probability is directly related to the area of the patches ( $i$ and $j)$ and inversely related to the distance between the centroids of each patch.

The next step is the calculation the "traversability" and recruitment values for each patch. Traversability is defined as the sum of the partial dispersal flux probabilities values for every link that is on at least one path connecting two given patches in the habitat. In this context, traversability is a measure of the permeability of a patch to infection coming from different patches in the network of the landscape. From the definition of traversability, the recruitment of a patch is a measure of the importance of that patch in the general network. The recruitment factor $R$ is defined as

$$
R=\sum_{i=1}^{m} S_{i} K_{i}
$$

where $S_{i}$ again is the size of patch $i, C S_{i}$ the $C S$ of that patch for ticks, and $m$ the number of patches directly linked to the patch under consideration. Therefore, recruitment is a value for each patch, computed from the sum of $K_{i}$ values and the local cluster of patches directly connected to the patch of concern.

The patch composition in the State of New York, in particular the vegetation component, was 
obtained from the land use and land cover (LULC) digital data set provided by the Geological Survey of United States. While this is a relatively old data set, superseded in some aspects by more modern digital data about landscape composition, it has a series of features of interest for this work which are absent in recent updates. Most important for this presentation is the resolution of the data, and the inclusion of several categories of vegetation of particular importance for the understanding of the factors regulating the spatial abundance of ticks.

\section{Model for risk index at patch level}

The importance of a combination of host species in the prevalence of infected I. scapularis nymphs has been previously reported (i.e. Ginsberg et al., 2005). These studies addressed questions about the importance of key hosts to sustain a proportion of infective nymphs, and demonstrate that the absence of some hosts will produce a sharp decrease in the number of infected nymphs. The current paper is based upon these previous findings and incorporates the preferences of the ticks towards natural hosts, which takes into account the ability of these animals to support particular portions of the tick population and their ability to infect these ticks (LoGiudice et al., 2003). Hosts included in the current model are Odocoileus spp., the white-footed mouse, the eastern chipmunk, the white-tailed deer, the raccoon, the Virginia opossum, the striped skunk, shrews, particularly the short-tailed shrew, Sorex spp., the red squirrel, the grey squirrel and special species birds (American robin, ovenbird, veery and wood thrush). Data on host presence and abundance used in the present paper have been obtained from the gap analysis project (GAP) which maps the habitats and the distribution of vertebrates in the United States at a very high resolution (http://gapanalysis.nbii.gov/portal/community/GAP_Analysis_Progr am/Communities/GAP_Home/). Data for birds and mammals in the State of New York were obtained and incorporated into a geographical information system (GIS) at the patch level as the number of host species reported to be present into a given vegetation patch. The recruitment obtained in the previous step, the preferences of nymphs to feed on a given host (in percentage), the moulting percentage after feeding on that particular host and the capacity of the host in question to infect the feeding tick (in percentage) were used to produce an IR index. Reported data about percentage of infected hosts are restricted to local studies with small or no temporal extensions. Because the lack of suitable data on host infection rates covering the State of New York it was assumed that the $50 \%$ of hosts in the region were infected and that tick prevalence on these hosts was homogeneous across the state.

It is implicitly assumed that the ticks use the hosts as available in the community of animals at a given vegetation patch and according to their abundance as provided by the GAP data. According to data by Giardina et al. (2000) on the preferences of immature ticks towards the different host species, the lack of one or more host species will drive the relocation of ticks to the remaining hosts present on such a vegetation patch. Admittedly, this is a model assumption that may be not real under all circumstances. However, it has been demonstrated that the lack of diversity in host communities tend to produce a high aggregation of ticks on the available hosts reducing the dilution effect (Ostfeld and Keesing, 2000).

The risk index was plotted and correlated at the patch level against host diversity and abundance derived from the raster layers of empirical data taking into account the measures of habitat connectivity and fragmentation mentioned above. In order to control the relationship between estimated IR values and the presence or absence of key hosts simple or multiple correlations were performed between IR and host density as well as between IR and recruitment together with host density. Multiple correlations between IR values and recruitment plus hosts density were also performed for selected patches at different densities of target hosts. Patches were selected according to the ratio between Peromyscus and other hosts (birds, squirrels and shrews). 
Comparison of risk index and actual prevalence rate of Lyme disease

While the model described here provides an estimation of the risk of infection from a tick bite at the patch level, it is hard to compare with actual infection rates in ticks. Consequently, it was decided to compare the spatial distribution of the risk of infection from a tick bite with the long-term infection prevalence rates in humans as reported for the different counties in the state. Rates in humans were obtained from the Department of Health of the State of New York (http://www.health.state.ny.us/statistics/chac/general/lyme.htm) and averaged for the years 2000-2007. It is not expected to find a straightforward relationship between IR and the prevalence of disease in humans, since these rates are reported according to the place of residence, while the human prevalence of infection depends upon a complex array of social factors such as tourism, picnic tours or other activities which brings people outside their place of residence. In addition, the general attractiveness of a given region or place also plays a role. All these factors cannot be quantified in a satisfactory way and our empirical validation is aimed only to provide a crude comparison between spatial distribution of risk and the rates of human disease due to Lyme borreliosis.

\section{Results}

The CS for I. scapularis is supposedly homogeneous in the State of New York but the model predicts maximum tick suitability for the centre with large areas of patches attractive for ticks, while the CS values decrease towards the western part of the state (Fig. 2). The climate layers providing an AUC of about 0.97 (maximum $=1$ ), point to winter temperatures and annual rainfall as the most restrictive variables for tick suitability. When details for habitat fragmentation were included to obtain values for tick recruitment (Fig. 3), the maximum was obtained in the eastern part of the study area, i.e. along the Hudson River. Recruitment was found to

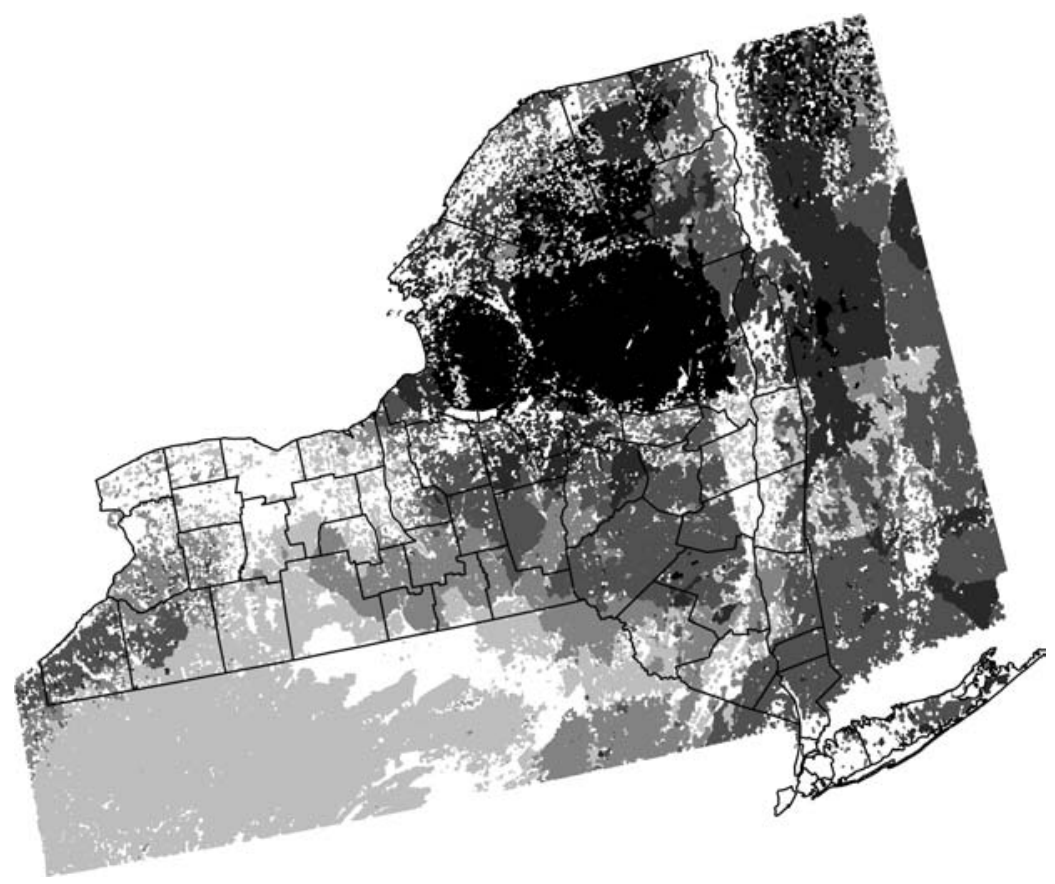

Fig. 2. The climate suitability for I. scapularis in the area of study, according to the MaxEnt algorithm and based upon the PRISM climate data-set for continental United States. The suitability range varies from null (white) to maximum (black). 


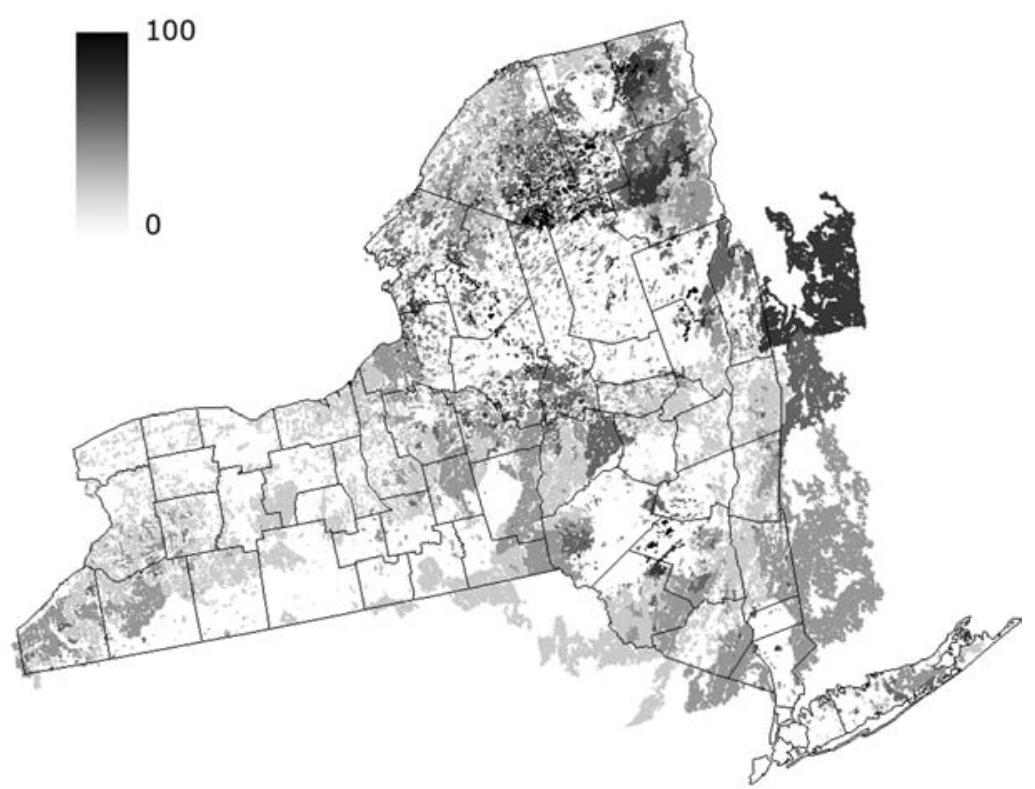

Fig. 3. Recruitment for I. scapularis in the study area calculated as described in materials and methods. Recruitment is a unit less index related with the density of ticks at every patch according to area, climate suitability and distance to near patches.

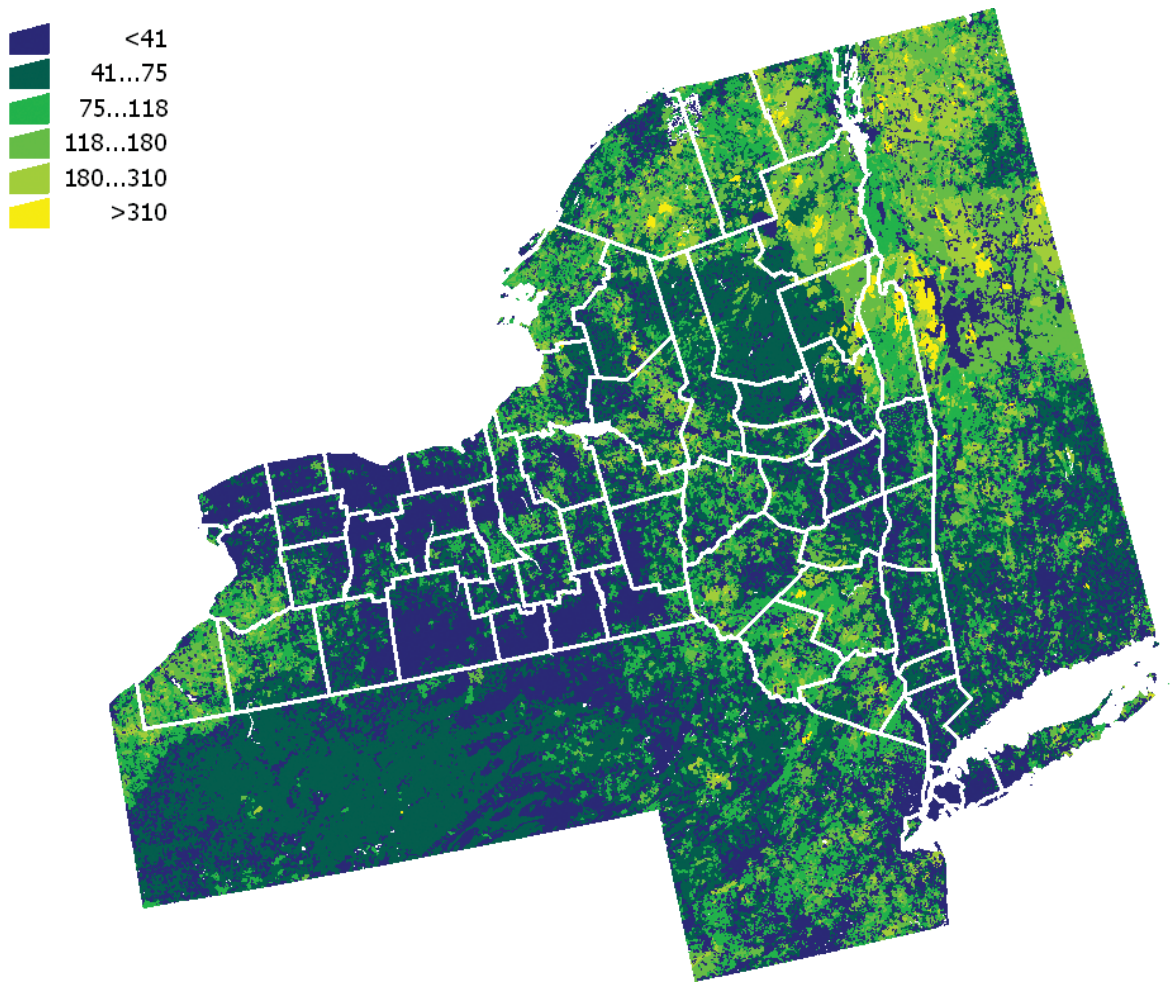

Fig. 4. Recruitment of infection (IR) for I. scapularis in the State of New York and surrounding areas. IR is dependent upon the density of infective hosts, the preferences of the tick towards each host species, and the recruitment of ticks at the patch level. 
be patchy across the state and generally lower in the northern and western counties.

The model predicts heterogeneous spatial IR distribution as a result of the combined distribution of tick recruitment and host density (Fig. 4). This figure further tells us that the areas with the highest rates are concentrated around the eastern and northern parts of the state with many small patches of high IR across the state. Clearly, there are large areas of low (but positive) IR values throughout the state, which are correlated with sites of low tick CS or with low abundance of one or several of the host species. Interestingly, there is a large zone characterized by many patches of very low IR in the western part of the state which could be a consequence of a combination of low CS and low recruitment. Figure 5 includes a summary of the densities of host species at patch level in order to permit visual comparison

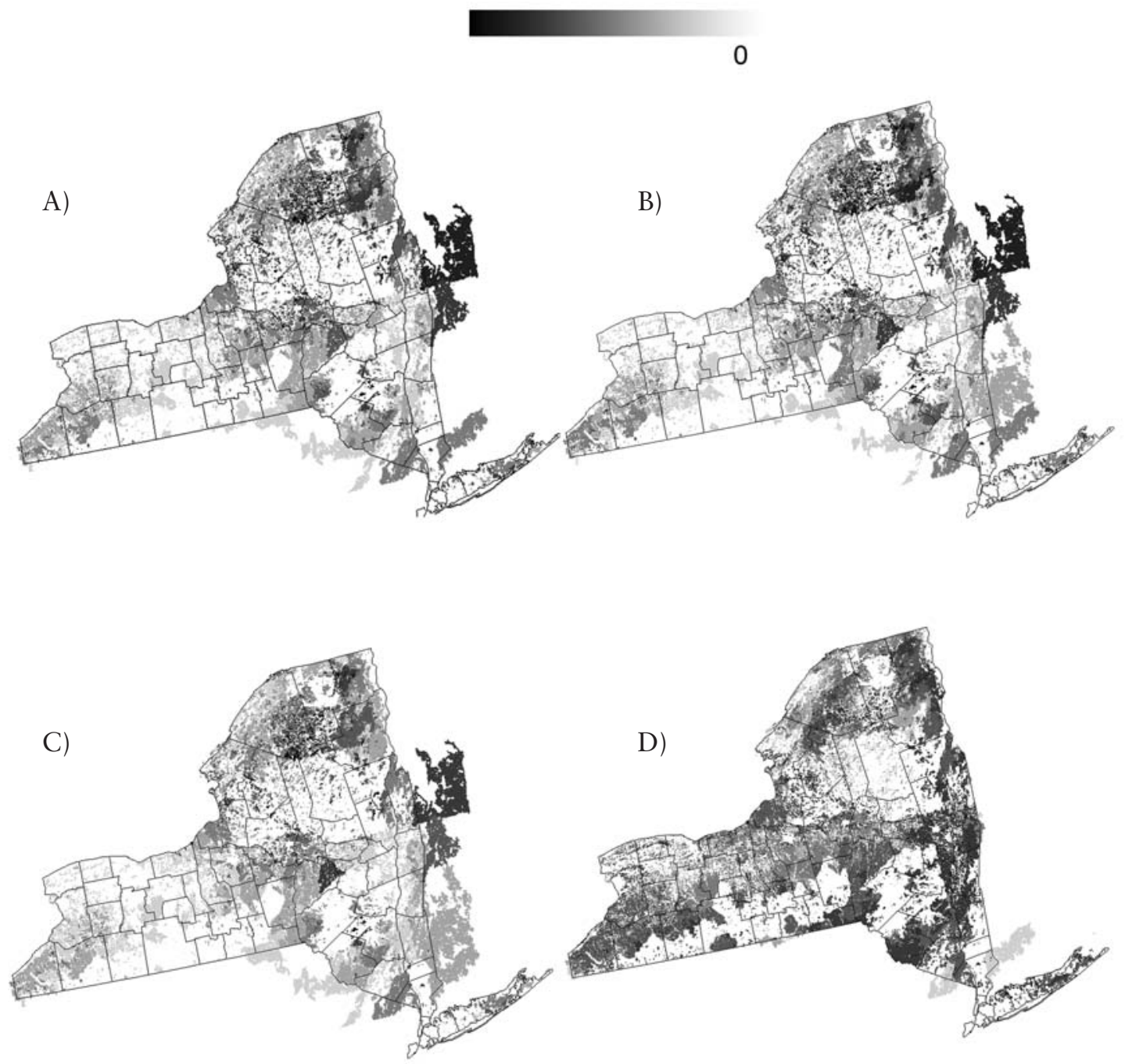

Fig. 5. Density per hectare for selected groups of hosts at the study area such as birds (A), Odocoileus virginianus (B), Peromyscus leucopus (C), and species of squirrels and shrews (D). The complete list of hosts included in the study is given in Material and Methods. Data are plotted according to estimates provided by the GAP project. Vegetation patches outside of the State borders are included when connected with the outer administrative limits. 
with the IR values in Fig. 4. The lineal correlation coefficients obtained from a comparison of the IR data with the densities of suitable hosts (Odocoileus, Peromyscus, several species of birds, squirrels and various shrews) are found in Table 1. This includes also multiple correlations of IR values against traversability and host density. The analysis was developed at the patch level. It is noteworthy that IR is not only well-correlated with the density of potentially important hosts in feeding and infecting tick populations, like deer and rodents, but also with the traversability and density of the putative key hosts for infecting the ticks (Peromyscus spp.) and for feeding them (Odocoileus spp.). Correlations obtained are clearly smaller for both birds and other groups of mammals. The best correlations were obtained when both recruitment and host density values were compared. Such direct comparisons between important epidemiological values constitute evidence of the importance of habitat fragmentation and the adequate proportion of the reservoir fauna. This is further supported by the fact that the IR values were not well-correlated with the relative density of hosts (Table 1 and Fig. 6). It should also be noted that sites with high densities of deer supported low relative numbers of

Table 1. Correlation coefficients between recruitment of infection (IR) as observed at every patch level in the State of New York and the density of different host groups (linear correlation) as well as between IR and traversability together with host densities (multiple correlations). All $r$ values are for $\mathrm{P}=0.001$.

\begin{tabular}{lcc}
\hline & $\begin{array}{c}\text { Correlation between } \\
\text { IR and host density }\end{array}$ & $\begin{array}{c}\text { Correlation between host } \\
\text { density and traversability }\end{array}$ \\
\hline Odocoileus spp. & 0.2357 & 0.9184 \\
Peromyscus spp. & 0.2362 & 0.9171 \\
Birds (several species) & 0.2324 & 0.6325 \\
$\begin{array}{l}\text { Others (including squirrels and several } \\
\text { species of shrews) }\end{array}$ & 0.2521 & 0.5922 \\
\hline
\end{tabular}
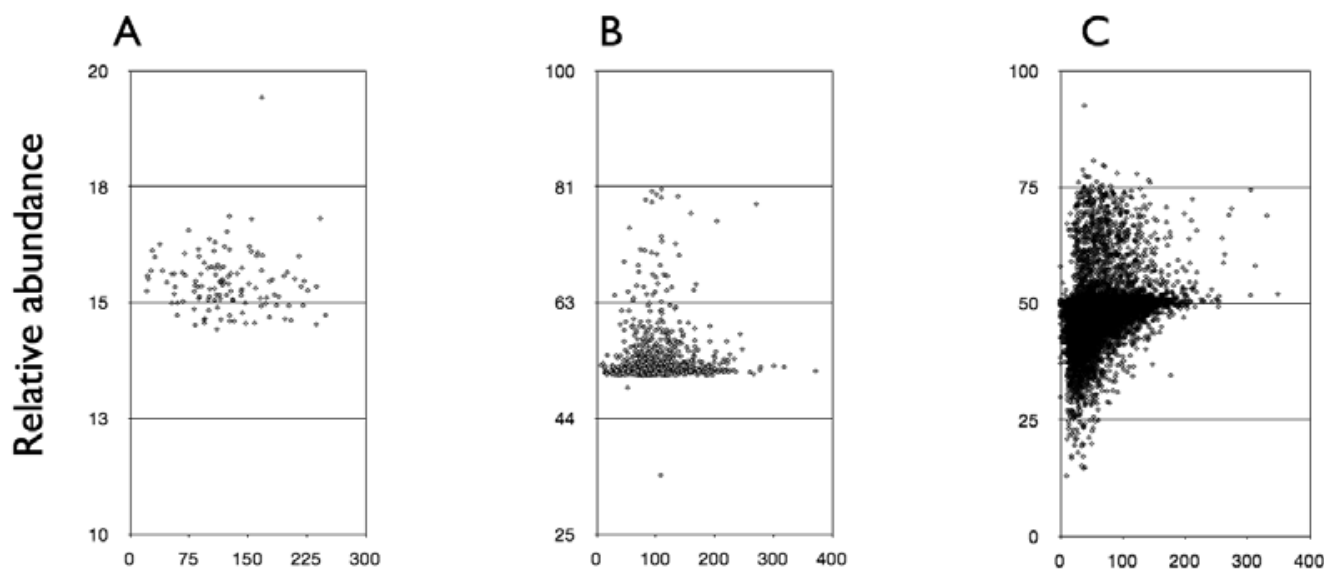

IR

Fig. 6. Plot of recruitment of infection (IR) as compared to the relative numbers of hosts species in areas with different densities of Odocoileus spp.: more than 5 specimens per hectare (A), between 2 and 5 specimens per hectare (B) and less than 2 specimens per hectare (C). The ratio between host species was calculated as the ratio between Peromyscus spp. densities and the rest of the hosts selected for this study. Therefore, a high value means that Peromyscus dominates, while a low value means a relative high representation of other hosts. 
Peromyscus reflected by very variable IR rates. However, sites with medium or low densities of deer have more variable numbers of this animal and allow therefore a better comparison. In this sense, there are no direct relationships between the abundance of Peromyscus hosts in relation to other types of hosts, and the computed IR rates. The rates of infection in humans in the different counties of the State of New York together with IR indexes obtained for each county are presented in Fig. 7. While there is no straightforward agreement between these series of values, counties reporting the highest rates in humans are also consistently associated with sites with higher IR values.

\section{Discussion}

In the forests of eastern North America, the immature stages of $I$. scapularis are extreme generalists, i.e. the feed on numerous different mammalian, avian and reptilian host species, most of which are believed, sometimes documented, to have low reservoir competence for Lyme disease. The white-footed mouse, which infects from $40 \%$ to $90 \%$ of the larval ticks that feed on it (Mather et al., 1989; Schmidt et al., 1999), has a wide tolerance for habitat variations. Therefore, species-poor communities tend to have mice and few other hosts, where- as species-rich communities have mice plus many other potential hosts. The latter should lead to a dilution of the impact of mice as they tend to rarely infect the feeding ticks. This would be an example of the Dilution Effect hypothesis, which predicts decreasing infection prevalence in the tick population with increasing host diversity (LoGiudice et al., 2008). We have previously demonstrated, both from empirical data and models, that the dilution effect is not only a consequence of host diversity, but also of the landscape composition and the preferences of hosts towards given vegetation features (EstradaPeña et al., 2008).

Different host species vary considerable with regard to their importance to pathogen transmission and the dynamics of infection (Brunner and Ostfeld, 2008). In this sense, the risk of infection for humans should be thought of as a function of the host community composition and the abundance of the ticks feeding on them. In the current study we used the concept of "reservoir potential" introduced by Mather et al. (1989) and empirically computed by Giardina et al. (2000) and LoGiudice et al. (2003) among others. Additional studies have been carried out by Ginsberg et al. (2005). However, to adequately describe the dynamics of pathogen transmission is a harder problem by several orders of magnitude. Previous reports, devoted to the study of

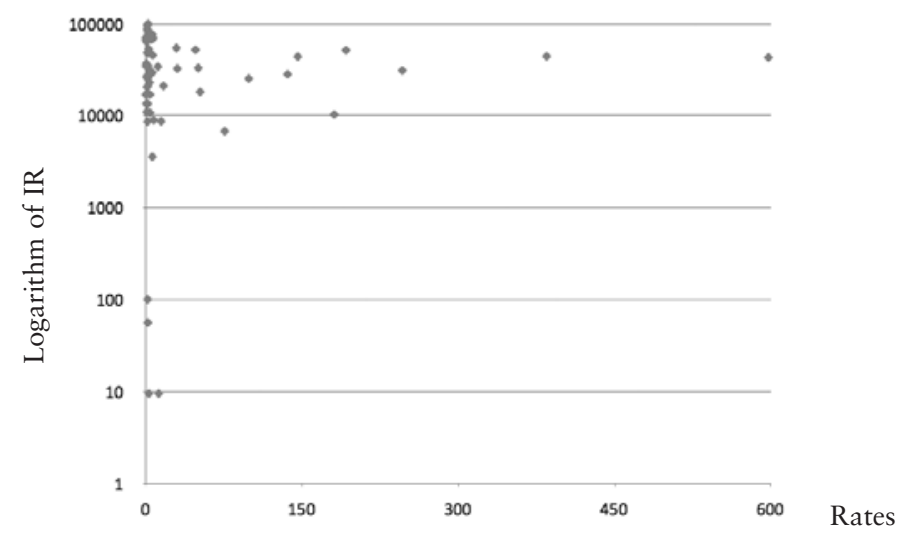

Fig. 7. Plot of the risk of infection as computed for each county in the State of New York and the reported Lyme disease rates in these counties, averaged for the period 2000-2008. 
the distribution of Borrelia infection based on theoretical frameworks (i.e. Madhav et al., 2004; Ogden et al., 2006), used a restricted set of hosts and addressed the relative importance of different hosts. In our model, we preferred to use a fixed set of hosts, with static rates of reservoir potential which may be quite different to the host used at every vegetation patch by the different stages of $I$. scapularis. Furthermore, our model has no temporal dimension and thus lacks the means to study the impact of variation of host density at given moments of the year, which may be an important variable for the infection rates for ticks (Ogden et al., 2005, 2008). One central assumption we make is that with the presence of high host densities, hosts of different species will divert ticks from each other. The true dynamics of this phenomenon remains largely unknown (Madhev et al., 2004).

Many previous studies have been applied to the study of the landscape relationships between the risk for Lyme disease and habitat patchiness. These studies have clearly demonstrated that the risk to acquire this infection is directly dependent upon a complex array of biological features such as tick density, presence and abundance of reservoir hosts, and degree of contact between the humans and infected ticks. Clearly, landscape fragmentation is a key factor influencing the rates of contact between people and infected ticks, while, on the other hand, the understanding of the relationship between landscape composition and tick abundance is still immature. While several models aim to explain the presence and/or abundance of ticks in an area due to CS, few have concentrated on the effects of landscape fragmentation as a direct driver of the abundance of ticks. Although researchers have previously developed useful models to understand the ecology of I. scapularis (i.e. Mount et al., 1997; Giardina et al., 2000), none have included a spatially explicit component except the one developed by Madhav et al. (2004). Some studies have used the framework used here to demonstrate how infection rates of wild ungulates (deer) with a tick-borne pathogen were strongly dependent upon the complex array of the patches in the landscape, as well as the abundance of ticks and host preferences towards a given vegetation patch. All these details were validated using empirical data (Estrada-Peña et al., 2008). However, the situation in the case of Lyme disease is far more complex. The wide variety of hosts, the extreme variability of habitat fragmentation and the many social conditions which influence the contact ratio between humans and pathogen-carrying ticks, produce conceptual frameworks out of the modelling attempts. Because we lack detailed state-wide surveys about the infection rates of $I$. scapularis ticks, we must to rely upon prevalence rates as derived from reports of the disease in humans. This is a serious but understandable drawback since these rates are dependent upon an extremely complex array of social factors which could not be included in this model. However, the high rates reported from the Long Island area provide an interesting demonstration of urban habitat where the IR is almost zero.

The main outcome of this study is the finding that habitat fragmentation is of pivotal importance for the variation of the rates of infected ticks in an area endemic for Lyme borreliosis debunking the idea that host composition is the predominant factor. As a consequence, we propose such a model as the theoretical framework for the application and comparison of empirical results about infection rates of ticks. While the dilution effect should be present at the patch level, the high fragmentation observed in the study area and the high traversability values found would in practice permit the exclusion of this effect in a spatial context. It is remarkable that the best correlations between risk for infection, traversability and hosts density were obtained for Peromyscus spp. and Odocoileus spp., which are considered to be the pivotal hosts for feeding and infecting ticks, respectively (Ostfeld et al., 2006), while lower correlation values were found for birds and other mammals, considered the main drivers of the dilution effect. Therefore, while obviously more dependent upon habitat fragmentation as measured by traversability, risk of infection should be diluted in the presence of a fauna acting as potential "dilu- 
tor" of the pathogen because they divert the ticks.

The importance of habitat fragmentation, tick abundance, and densities of some target hosts have been stressed as key factors in the risk of Lyme disease for humans (i.e. Guerra et al., 2002). A simple relation between human infection rates and IR can be foreseen from the results presented here. Our results are consistent (within the obvious error derived from the use of human prevalence rates at the county scale) with the pattern of infection observed in the state, i.e. the large area of high IR along the Hudson River, itself a communication artery which is further strengthened by the concentration of main roads. Thus, social factors link the most populated areas in the state with high-risk patches through the dense network of roads along the eastern part of the state. The highest rates of prevalence are consistently reported for southern portions of the state which has the highest population densities as well as an IR close to zero. This suggests that human-derived prevalence rates are less related to the presence of active disease foci near the home but more strongly to social factors such as trekking, picnics, etc. in other areas characterized by scattered residences in a highly fragmented landscape. However, infection rates cannot be obtained from pure combinatory relationships between host abundance in a straightforward way, since they strongly depend upon habitat relationships. Thus, a simple infection index can neither be built on the faunal composition and its relative numbers nor on tick recruitment estimates alone.

\section{Acknowledgements}

The author wish to thank Dr. Lance A. Durden (Georgia Southern University) for providing a large set of records of Ixodes scapularis in the USA stored in the U.S. National Tick Collection, Statesboro, GA, USA. The climate PRISM model data, created in March 2008, came from the PRISM Group, Oregon State University (http://www.prismclimate.org). Land use and land cover data are copyrighted by the U.S. Geological Survey, under the title "USGS Land Use and Land Cover (LULC) Data", released in 1994. GAP animal distribution data come from regional- and state-level projects and are coordinated by the USGS Biological Resources Division. It is a cooperative effort among regional, state and federal agencies, and private groups for inventory, monitoring, research and information transfer.

\section{References}

Brunner JL, Ostfeld RS, 2008. Multiple causes of variable tick burdens on small-mammal hosts. Ecology 89, 22592272.

Drew ML, Loken KI, Bey RF, Swiggum RD, 1988. Ixodes dammini: occurrence and prevalence of infection with Borrelia spp. in Minnesota. J Wildl Dis 24, 708-710.

Estrada-Peña, A, 2002. Increasing habitat suitability in the United States for the tick that transmits Lyme disease: a remote sensing approach. Environ Health Perspect 110, 635-640.

Estrada-Peña A, 2003. The relationships between habitat topology, critical scales of connectivity and ticks abundance Ixodes ricinus in a heterogeneous landscape in northern Spain. Ecography 26, 661-673.

Estrada-Peña A, Acevedo P, Ruiz-Fons F, Gortazar C, de la Fuente J, 2008. Evidence of the importance of host habitat use in predicting the dilution effect of wild boar for deer exposure to Anaplasma spp. PLoS ONE 3, e2999.

Estrada-Peña A, Martinez JM, Sanchez Acedo C, Quilez J, Del Cacho E, 2004. Phenology of the tick, Ixodes ricinus, in its southern distribution range (central Spain). Med Vet Entomol 184, 387-397.

Giardina AR, Schmidt KA, Schauber EM, Ostfeld RS, 2000. Modeling the role of songbirds and rodents in the ecology of Lyme disease. Can J Zool 78, 2184-2197.

Ginsberg HS, Buckley PA, Balmforth MG, Zhioua E, Mitra S, Buckley FG, 2005. Reservoir competence of native North American birds for the Lyme disease spirochete, Borrelia burgdorferi. J Med Entomol 42, 445-449.

Guerra M, Walker E, Jones M, Paskewitz S, Cortinas R, Stancil A, Beck L, Bobo M, Kitron U, 2002. Predicting the risk of Lyme disease: habitat suitability for Ixodes scapularis in the North Central United States. Emerg Infect Dis 8, 289-297.

Kalluri S, Gilruth P, Rogers D, Szczur M, 2007. Surveillance of arthropod vector-borne infectious diseases using remote 
sensing techniques: a review. PLoS ONE 3, e116.

Kitron U, Jones CJ, Bouseman JK, Nelson JA, Baumgartner DL, 1992. Spatial analysis of the distribution of Ixodes dammini (Acari: Ixodidae) on white-tailed deer in Ogle County, Illinois. J Med Entomol 29, 259-266.

LoGiudice K, Duerr ST, Newhouse MJ, Schmidt KA, Killilea ME, Ostfeld RS, 2008. Impact of host community composition on Lyme disease risk. Ecology 89, 2841-2849.

LoGiudice K, Ostfeld RS, Schmidt KA, Keesing F, 2003. The ecology of infectious disease: effects of host diversity and community composition on Lyme disease risk. Proc Natl Acad Sci USA 100, 567-571.

Madhav N, Brownstein JS, Tsao JI, Fish D, 2004. A model for the range expansion of blacklegged tick (Acari, Ixodidae). J Med Entomol 41, 842-852.

Mather TN, Wilson ML, Moore SI, Ribeiro JMC, Spielman A, 1989. Comparing the relative potential of rodents as reservoirs of the Lyme disease spirochete (Borrelia burgdorferi). Am J Epidemiol 130, 143-150.

Mount GA, Haile DG, Daniels E, 1997. Simulation of management strategies for the blacklegged tick (Acari: Ixodidae) and the Lyme disease spirochete, Borrelia burgdorferi. J Med Entomol 34, 672-683.

Novak MG, Rowley WA, Platt KB, Bartholomew DM, Senne M, 1991. Ixodes dammini (Acari, Ixodidae) and Borrelia burgdorferi in Iowa. J Iowa Acad Sci 98, 99-101.

Ogden NH, Bigras-Poulin M, Hanincová K, Maarouf A, O'Callaghan CJ, Kurtenbach K, 2008. Projected effects of climate change on tick phenology and fitness of pathogens transmitted by the North American tick Ixodes scapularis. J Theor Biol 3, 621-632.

Ogden NH, Bigras-Poulin M, O'Callaghan CJ, Barker IK, Lindsay LR, Maarouf A, Smoyer-Tomic KE, Waltner-Toews D, Charron D, 2005. A dynamic population model to inves- tigate effects of climate on geographic range and seasonality of the tick Ixodes scapularis. Int J Parasitol 35, 375-389. Ogden NH, Maarouf A, Barker IK, Bigras-Poulin M, Lindsay LR, Morshed MG, O'callaghan CJ, Ramay F, WaltnerToews D, Charron DF, 2006. Climate change and the potential for range expansion of the Lyme disease vector Ixodes scapularis in Canada. Int J Parasitol 36, 63-70.

Ostfeld RS, Canham CD, Oggenfuss K, Winchcombe RJ, Keesing F, 2006. Climate, deer, rodents, and acorns as determinants of variation in Lyme-disease risk. PLoS Biol 4, e145.

Ostfeld RS, Keesing F, 2000. Pulsed resources and community dynamics of consumers in terrestrial ecosystems. Trends Ecol Evol 15, 232-237.

Phillips SJ, Anderson RP, Schapire RE, 2006. Maximum entropy modeling of species geographic distributions. Ecol Modell 190, 231-259.

Randolph SE, 1998. Mighty theories from little acorns grow: is Lyme disease risk predictable from mast-seeding oak trees? Trends Ecol Evol 13, 301-303.

Schauber EM, Ostfeld R, Evans AS, 2005. What is the best predictor of annual Lyme disease incidence: weather, mice or acorns? Ecol Appl 15, 575-586.

Schmidt KA, Ostfeld RS, Schauber EM, 1999. Infestation of Peromyscus leucopus and Tamias striatus by Ixodes scapularis (Acari: Ixodidae) in relation to the abundance of hosts and parasites. J Med Entomol 36, 749-757.

Schulze TL, Jordan RA, Hung RW, 1998. Comparison of Ixodes scapularis (Acari: Ixodidae) populations and their habitats in established and emerging Lyme disease areas in New Jersey. J Med Entomol 35, 64-70.

Swets JA, 1988. Measuring the accuracy of diagnostic systems. Science 240, 1285-1293.

Urban D, Keitt T, 2001. Landscape connectivity: a graphtheoretic perspective. Ecology 82, 1205-1218. 